\title{
THE REACTION OF PHOSPHORUS-CONTAINING INTRACELLULAR INCLUSIONS TO SPACE WEATHER CHANGES
}

\author{
Gromozova E.N., Kachur T.L., Voychuk S.I., Kharchuk M.S. \\ Danylo Zabolotny Institute of Microbiology and Virology of NAS of Ukraine, Kiev \\ gren.elen@gmail.com
}

\begin{abstract}
It is known that the bio-astronomical effect of Chizhevsky-Velhover is based on the cytochemical metachromatic reaction of polyphosphatecontaining volutin granules of microorganisms. As a result of long-term monitoring (2001-2015 years) of this of cytochemical index in yeasts Saccharomyces cerevisiae, it was shown that metachromatic staining of the granules possessed rhythmicity and connection with space weather. Under laboratory conditions the change of metachromasy was achieved by applying different stress factors: anaerobiosis, low temperature, acidity. Simulation of metachromatic staining in vitro by using solutions of inorganic polyphosphate with other compounds showed that this reaction was possible for the low concentrations of these polymers. The effect of the low concentrations was more expressed with increasing chain length of polyphosphate. The inhibition of the metachromasy in solution with added protein and calcium chloride indicated that this of reaction involved the presence of the protonated sites in the polymers. It is assumed that metachromatic staining of volutin granules in vivo may depend on other conditions including conformational restructuring of a polymer or sol-gel phase transitions. Thus, the question whether the metachromatic reaction of intracellular polyphosphate granules to the space weather changes is driven by a response to associated stress or despite the apparent similarity of the effects they are of a different nature remains unclear.
\end{abstract}

Key words: space weather, polyphosphate, volutin granules, bio-astronomical effect, metachromatic reaction

\section{Introduction}

To date, there is a significant amount of data about the influence of space weather on the biological processes. The connection between the behavior of microorganism and cosmophysical factors was reflect in bio-astronomical effect of Chizhevsky-Velhover [1]. reflected in bio-astronomical effect of Chizhevsky-Velhover [1]. This effect is based on the metachromatic reaction of volutin granules stained with basic dyes such as (methylene and toluidine blue) [2]. Volutin granules are intracellular structures which widely spread among microorganisms both prokaryote and eukaryote. Inorganic polyphosphates are the main component of granules. These polymers are known to perform different functions depending on localization in the cell $[3,4]$. It is assumed that one of these functions can be a reception of the cosmophysical signals [5]. Inorganic polyphosphates, so- called "fossil molecules", are the most ancient molecules arisen in inanimate nature and accompanied biological objects at all stages of evolution [3].The formation of evolutionary and adaptive scenario of ancient biosystems functioning was, first of all, under the action of helio- and geophysical factors. So, we can assume that variation of these conditions took part in the formation of rhythmic biological processes with the participation of polyphosphates. Thus, investigation of the inorganic polyphosphate features of volutin granules manifested using metachromatic staining may bring us closer to understanding of the mechanisms of the biotropic cosmic weather action.

The objective of the present work was to research the effects of the stress factors on the metachromatic reaction of volutin granules and its simulation in vitro.

\section{Methods}

In the experiments, the yeasts Saccharomyces cerevisiae Y-517 from Ukrainian Collection of Microorganisms of Danylo Zabolotny Institute of Microbiology and Virology of NAS of Ukraine were used. Cultures were grown on the wort agar for 24 hours at temperature $28^{\circ} \mathrm{C}$. Everyday in set time (12:00 Kiev time), cells were stained with methylene blue according to Loeffler for determining the presence of the metachromatic reaction [5]. Simultaneously, they were subcultured on the fresh nutrient medium. Assessment of the metachromatic reaction was performed by stained smears in light field using light microscopy (microscope "PrimaStar", Zeiss). Three-point system assessment was used, where "1" - absence of metachromasy (blue color of the granules), "2" and "3" - different degree of expressing metachromasy (violet and violet-red colour of the granules, respectively). Stress conditions were low temperature $\left(-4^{0} \mathrm{C}\right)$, anaerobiosis and acidity $(150 \mathrm{mM}$ acetic acid).We prepared solutions of inorganic polyphosphates with chain length 18 and 200 residues of phosphoric acid at a final concentration of 0.025 to 5 $\mathrm{mg} / \mathrm{ml}$. $\mathrm{pH}$ was adjusted to neutral values by titration of 1 $\mathrm{N}$ solution of $\mathrm{NaOH}$. Concentration of methylene blue in test samples was $0.005 \mathrm{mg} / \mathrm{ml}$. Glucose $(0.01,0.1$ and $1 \%$ ), calcium chloride $(1,15$ and $30 \mathrm{mM})$ and bovine serum albumin (BSA; 0.1, 0.5 and $1 \mathrm{mg} / \mathrm{ml}$ ) were added to polyphosphate solutions. The uptake spectra of methylene blue in the samples wereevaluated usingthe spectrophotometer DU-8B (Backman, USA) with water as a blank sample. Data was treated statistically by program of Statistica 6.0 (StatSoft, Inc. 2002). 


\section{Results and discussion}

As a result of long-term monitoring which was conducted from 2001 to 2015 years it was found that the metachromatic reaction of volutin granules of $S$. cerevisiae yeast had rhythmicity and connection with space weather [6]. Rhythm of metachromasy almost completely coincided with the known space periods. This coincidence could mean conjugation of rhythms or synchronization of biological rhythms with cosmophysical variations but it has not a causal relationship [7]. Except the presence of rhythmicity statistically reliable correlation of this reaction with whole range of cosmophysical factors at the same period [6] could be the evidence of connection of metachromasy with space weather.

In our experimental studies under laboratory conditions the metachromatic reaction of volutin granules of the yeast cells was observed when using different stress factors: low temperature, anaerobiosis and acidity. . Full colour change of volutin granules to violet-red ("3") was observed at low temperature and acidity (see Fig. A and B), whereas at anaerobiosis it was marked $90 \%$ of cases with such colouring (see Fig. C). Thus, the changes of the phosphoric metabolism of the cell might probably take took place as a result of the stress impact, and were reflected in the appearance of metachromatic staining of volutin granules.

\section{Control}
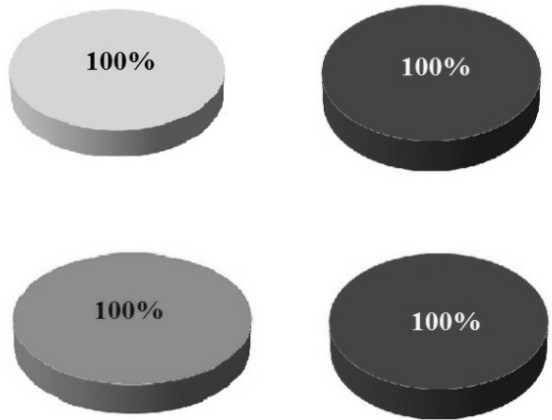

B
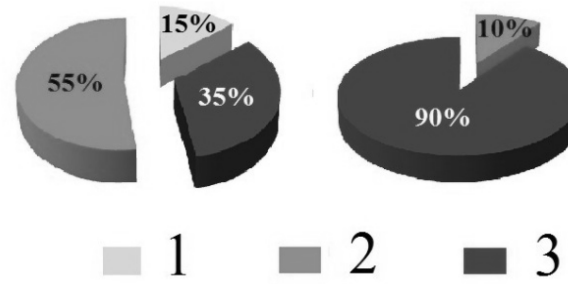

\section{Experiment}

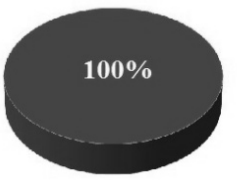

A

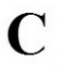

Figure 1: Impact of stress factors on the metachromatic reaction: $\mathrm{A}-$ low temperature $\left(-4^{\circ} \mathrm{C}\right) ; \mathrm{B}-$ acidity $(0,15$ $\mathrm{M})$; C - anaerobiosis; 1 - absence of metachromasy (blue colour of granules); 2 - weakly expressed metachromasy (violet colour of granules); 3 - well expressed metachromasy (violet-red colour of granules).

The simulation of the metachromatic reaction in vitro with polyphosphate solutions in combination with other compounds, showed that protein and calcium chloride inhibited aggregation of methylene blue on a polymer. Glucose, in turn, did not interfere with the appearance of the metachromatic staining. Thus, inhibition of the metachromatic staining by, adding protein and calcium chloride to the solution indicated that this reaction took place in the presence of the protonated sites in the polymers. It was shown that (Fig. 2) the metachromatic shift in the spectrum of uptake of the dye was caused by a decrease of polyphosphate concentration (of $0.1 \mathrm{mg} / \mathrm{ml}$ to $0.025 \mathrm{mg} / \mathrm{ml}$ ). The effect of the low concentrations was more expressed with increasing chain length of polyphosphate (Fig. 2 B).

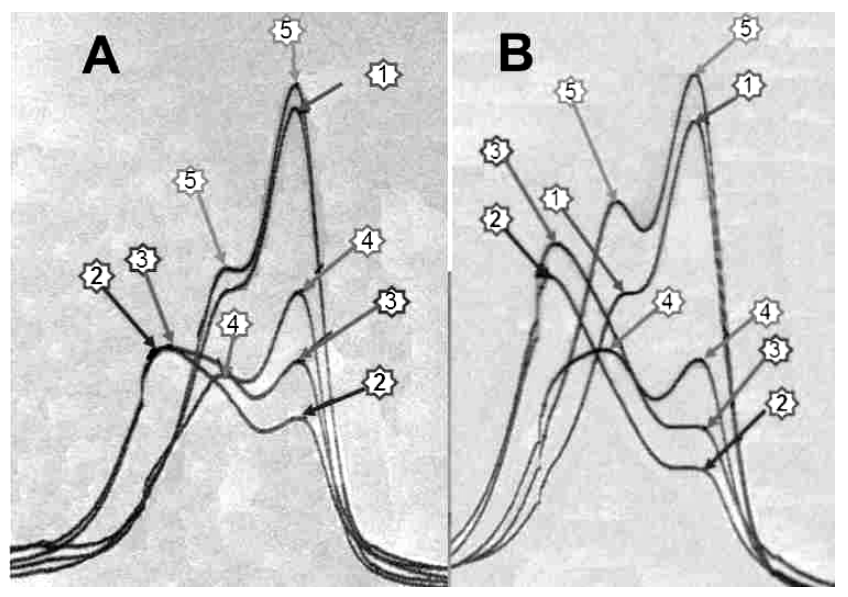

Figure 2: The uptake spectra of the methylene blue solution $(0.005 \mathrm{mg} / \mathrm{ml})$ and its complexes with polyphosphates. Polyphosphates chain lengths: 18 (A) and 200 (B) of phosphoric residues at concentrations: (1) 0 (control); (2) 0.025 ; (3) 0.1 ; (4) 0.4 ; (5) 2.5 (mg/ml).

The cause of the metachromatic staining is known be aggregation of the dye on the polyanions serving as a matrix [8]. Polyphosphates are one of such polyanionic compounds. A metachromasy does not occur if polyphosphates have chain length less than 10 residues of phosphoric acid [9]. As these polymers are known to be actively involved in the antistress reactions of microorganisms [10] the metachromasy of volutin granules can be assumedly caused by stress impact of the cosmophysical events. However, the reaction to the stress factors usually is accompanied by the polyphosphate accumulation [10], which should not cause metachromatic staining of the polyphosphate-containing structures. Thus, the processes causing metachromasy in vivo differed from those which took place in vitro. It is assumed that this reaction might be also caused by the physico-chemical factors conformational changes of a chain, where the distance between the reaction groups is 5 and less $\AA$ [11], or sol-gel phase transitions.

Thus, the question whether the metachromatic reaction of intracellular polyphosphate granules to the space weather changes is driven by a response to associated stress or despite the apparent similarity of the effects they are of a different nature remains unclear.

\section{References}

1. A quick reference guide to space biology and medicine. Moscow: Medicine, 1967 (in russian).

2. Serafim L.S., Lemos P.C., Levantesi C., Tandoi V., Santos H., Reis M.A.M.: 2002, Journal of Microbiological Methods, 51, 1-18. 
3. Brown M.R.W., Kornberg A.: 2004, PNAS, 101, №46, 16085.

4. Lichko L., Kulakovskaya T., Pestov N., Kulaev I.: 2006, Biosci. Rep., 26, 45.

5. Practicum for microbiology: tutorial guide /ed. Egorov N.S., Moscow: Moscow University Press, 1976, 307 p. (in russian).

6. Gromozova E.N., Grigoriev P.E., Kachur T.L., Voychuk S.I.: 2010, Biophysical processes and Biosphere, 9, No 2, 67 (in russian).

7. Vladimirsky B.M. Impact of solar activity on biospherenoosphere (Heliobiology from Chizhevsky A.L. to the present days) /B.M.Vladimirsky, N.A.Temurjyants, M: Publishing house, 2000, 374 p. (in russian).

8. Shirai M., Nagatsuka T., Tanaka M.: 1977, Micromol. Chem., 178, 37.

9. Lorenz B., Schröder H.C.: 1999, Inorganic Polyphosphates. Biochemistry, Biology, Biotechnology, Springer, 217 (in russian).

10. Ault-Riche D., Fraley C.D., Tzeng C.-M., Kornberg A.: 1998, Journal of Bacteriology, 180, No7, 1841.

11. Pierce E. Histochemistry theoretical and applied /ed. E. Pearce, Moscow: Univ. foreign. literature, 1962, 962 p. (in russian). 